\title{
Scalar curvature of definable CAT-spaces
}

Bernig, A

\begin{abstract}
We study the scalar curvature measure for sets belonging to o-minimal structures (e.g. semialgebraic or subanalytic sets) from the viewpoint of metric dierential geometry. Theorem: Let $\mathrm{S}$ be a compact connected definable pseudo-manifold with curvature bounded from above, then the singular part of the scalar curvature measure is non-positive. The topo- logical restrictions cannot be removed, as is shown in examples.
\end{abstract}

DOI: https://doi.org/10.1515/advg.2003.003

Posted at the Zurich Open Repository and Archive, University of Zurich ZORA URL: https://doi.org/10.5167/uzh-21845

Journal Article

Published Version

Originally published at:

Bernig, A (2003). Scalar curvature of definable CAT-spaces. Advances in Geometry, 3(1):23-43.

DOI: https://doi.org/10.1515/advg.2003.003 


\title{
Scalar curvature of definable CAT-spaces
}

\author{
Andreas Bernig \\ (Communicated by C. Scheiderer)
}

\begin{abstract}
We study the scalar curvature measure for sets belonging to $o$-minimal structures (e.g. semialgebraic or subanalytic sets) from the viewpoint of metric differential geometry. Theorem: Let $S$ be a compact connected definable pseudo-manifold with curvature bounded from above, then the singular part of the scalar curvature measure is non-positive. The topological restrictions cannot be removed, as is shown in examples.
\end{abstract}

\section{Introduction}

1.1 Plan of the paper and main results. One of the most important and most difficult problems in subanalytic geometry is to understand the induced length metric of subanalytic sets. For instance, the behavior of geodesics remains completely mysterious. Apart from a theoretical interest, this question has applications even outside mathematics, e.g. in robotics. This paper is devoted to the study of subanalytic sets by means of integral geometry and metric differential geometry.

In a preceding paper ([2]), we defined a scalar curvature measure for singular spaces. More precisely, we associated to each compact connected set belonging to some $o$-minimal structure (e.g. semialgebraic or subanalytic sets) a signed measure, called scalar curvature measure, which shares many of the properties of the usual scalar curvature of Riemannian manifolds. One of the main results of [2] was to relate the scalar curvature measure to curvature bounds in the sense of metric differential geometry. It has been shown that a lower bound $\kappa$ on the curvature of a compact connected definable set of dimension $m$ implies the lower bound $\kappa m(m-1) \operatorname{vol}(-)$ for the scalar curvature measure. This theorem generalizes in a non-trivial way the easy fact from differential geometry that positive sectional curvature implies positive scalar curvature.

In this paper, we carry on the study of scalar curvature measure of definable sets. We will show that the analogous result in the case of negative sectional curvature is valid under some minor topological restrictions.

The main theorem is the following (see 1.2 for definitions):

Theorem 1.1 (Main Theorem). Let $S$ be a compact connected definable pseudo-manifold with curvature bounded from above, then the singular part of $\operatorname{scal}(S,-)$ is non-positive. 
Remarks. If the dimension of $S$ is $m$ and the upper curvature bound $\kappa$, then we get an inequality between signed measures:

$$
\operatorname{scal}(S,-) \leqslant \kappa m(m-1) \operatorname{vol}(S,-) .
$$

This generalizes the classical upper bound for the scalar curvature $s \leqslant \kappa m(m-1)$ on $m$-dimensional Riemannian manifolds with sectional curvature bounded from above by $\kappa$.

If $S$ is a pseudo-manifold, then there exists a stratification with the property that each $m-1$-stratum is contained in the boundary of exactly two $m$-strata. This is what we really need.

The topological assumption made in the theorem is in general necessary. For instance, take the closed unit ball in Euclidean space. Being convex, it has non-positive curvature in the metric sense, but its total scalar curvature is strictly positive because of the boundary contribution. Another example will be presented in Section 4.

Since everything is done locally, the theorem remains true for closed connected definable sets $S$. In this case, $\operatorname{scal}(S,-)$ is still a difference of non-negative Borel measures and $\operatorname{scal}(S, U)$ is well-defined for precompact $U$.

The paper is organized as follows. After giving the basic definitions, we will show some results about geodesics on definable spaces, which are of independent interest, see Section 2. The proof of the main theorem is contained in Section 3. It consists of two steps, one for strata of codimension 1, one for strata of codimension 2 . The proofs in these cases are different, although they share some common features, as the use of the triangle inequality for angles and the extension property for geodesics. A consequence of one of the obtained formulas is the invariance of the scalar curvature measure under isometries. Such a result (actually a more general version) was conjectured by $\mathrm{J}$. Fu. This invariance property is obtained in Section 4. It is the generalization of the classical fact that the scalar curvature on a Riemannian manifold is an inner quantity.

The idea behind the proof of the main theorem is to use the fact that, in the situation we will consider, each geodesic can be extended beyond its endpoints, by properties of CAT-spaces. In codimension 2, the triangle inequality for angles then yields that the density at each point of the stratum is bounded from below by 1 . In codimension 1, a careful investigation of geodesics and angles between them is necessary. Again, we will conclude by the triangle inequality for angles that the scalar curvature measure is non-positive.

Acknowledgments. I would like to thank Professor Ludwig Bröcker for helpful discussions and his encouragement and the referee for useful comments.

1.2 Recall of definitions. First, we will introduce spaces with curvature bounded from above and give some properties that will be needed in the following proof. There is an excellent book, [4], where the reader can find a systematic treatment of spaces with curvature bounded from above. The following definitions are taken from Part II of that book. 
Definition 1.2. A geodesic triangle in an inner metric space consists of three points $A, B, C$ and geodesic segments $[A, B],[B, C]$ and $[C, A]$ between them. We say that it satisfies the $\operatorname{CAT}(\kappa)$ inequality $(\kappa \in \mathbb{R})$, if for all points $P \in[A, B]$ and $Q \in[A, C]$, we have $d(P, Q) \leqslant d(\tilde{P}, \tilde{Q})$, where $\tilde{P}$ and $\tilde{Q}$ are points on the sides $[\tilde{A}, \tilde{B}]$ respectively $[\tilde{A}, \tilde{B}]$ of a comparison triangle $\tilde{A}, \tilde{B}, \tilde{C}$ in the 2-dimensional space form $M_{\kappa}$ with constant curvature $\kappa$ with $d(\tilde{A}, \tilde{P})=d(A, P), d(\tilde{A}, \tilde{Q})=d(A, Q)$.

A metric space is called $d$ geodesic if all pairs of points a distance less than $d$ apart can be joined by a (not necessarily unique) geodesic.

We denote by $D_{\kappa}$ the diameter of $M_{\kappa}$, that is $D_{\kappa}=\infty$ for $\kappa \leqslant 0$ and $D_{\kappa}=\frac{\pi}{\sqrt{\kappa}}$ for $\kappa>0$.

Definition 1.3. a) An inner metric space $X$ is called $\operatorname{CAT}(\kappa)$ space if it is a $D_{\kappa}$ geodesic space and all geodesic triangles of perimeter less than $2 D_{\kappa}$ satisfy the CAT $(\kappa)$ inequality.

b) A metric space $X$ is said to be of curvature $\leqslant \kappa$ if it is locally a $\mathrm{CAT}(\kappa)$-space, i.e. for every $x \in X$ there exists $r_{x}>0$ such that the ball $B\left(x, r_{x}\right)$, endowed with the induced metric, is a $\mathrm{CAT}(\kappa)$-space.

Remark 1.4. The initials C, A, T stand for Cartan, Alexandrov and Toponogov.

Recall that in a metric space $X$, the angle $\angle(A, B, C)$ is defined as the angle at $\tilde{A}$ of a comparison triangle $\tilde{A}, \tilde{B}, \tilde{C}$ in Euclidean space. The Alexandrov angle between two geodesics $c, c^{\prime}$ with $c(0)=c^{\prime}(0)=x$ is defined as $\angle\left(c, c^{\prime}\right):=$ $\lim \sup _{t, t^{\prime} \rightarrow 0} \angle\left(x, c(t), c^{\prime}(t)\right)$.

The next few statements are taken from [4]. We will need them in the course of the proof of our main theorem.

Proposition 1.5. Suppose $X$ is a $\mathrm{CAT}(\kappa)$-space. Then:

- The Alexandrov angle between the sides of any geodesic triangle of perimeter smaller than $2 D_{\kappa}$ in $X$ with distinct vertices is not greater than the angle between the corresponding sides of its comparison triangle in $M_{\kappa}^{2}$. Moreover, every $D_{\kappa}$-geodesic metric space with this property is actually a $\mathrm{CAT}(\kappa)$-space.

- Every local geodesic in $X$ of length at most $D_{\kappa}$ is a geodesic.

- If $X$ is a topological manifold, then each geodesic $\gamma:[a, b] \rightarrow X$ can be extended to a geodesic $\gamma:[a-\varepsilon, b+\varepsilon] \rightarrow X$ for some $\varepsilon>0$. It follows that, if $X$ is complete, any geodesic can be extended to a geodesic of length $D_{\kappa}$.

Next, we recall the definition of $o$-minimal structures. Most propositions about semialgebraic sets can be deduced from a short list of properties, including the TarskiSeidenberg principle (which states that the projection of a semialgebraic set is semialgebraic). Each class of sets satisfying these axioms shares automatically many useful properties with semialgebraic sets, e.g. boundedness of number of connected components. The definition is the following: 
Definition 1.6. An $o$-minimal structure is a sequence $\sigma=\left(\sigma_{n}\right)_{n=1,2,3, \ldots}$ such that:

a) $\sigma_{n}$ is a Boolean algebra of subsets of $\mathbb{R}^{n}$.

b) Algebraic subsets belong to $\sigma$.

c) If $S \in \sigma_{n}$ and $S^{\prime} \in \sigma_{m}$, then $S \times S^{\prime} \in \sigma_{n+m}$.

d) If $S \in \sigma_{n+1}$ then $\pi(S) \in \sigma_{n}$ where $\pi: \mathbb{R}^{n+1} \rightarrow \mathbb{R}^{n}$ is the projection on the first $n$ coordinates.

e) $\sigma_{1}$ consists exactly of finite unions of points and intervals.

Examples 1.7. The smallest example of an $o$-minimal structure is the set of semialgebraic sets.

Globally subanalytic sets form an o-minimal structure. A set is called globally subanalytic if its closure in the projective space is subanalytic.

Sets definable over $\mathbb{R}_{\exp }=(\mathbb{R},+, \cdot,<, \exp )$ yield another example of an $o$-minimal structure.

The basic reference for $o$-minimal structures is [21], see also [10]. In the rest of the paper, we fix an $o$-minimal system and refer to its elements as definable sets.

Definition 1.8. Let $S \subset \mathbb{R}^{n}$ be a compact connected definable set. Then the restriction of the Euclidean metric of $\mathbb{R}^{n}$ defines a metric on $S$, also called Euclidean metric and denoted by $d_{e}$. In general, $d_{e}$ is not a length metric, but it induces a unique length metric on $S$, which we will denote by $d_{i}$. It is called inner metric of $S$.

Let $\delta\left(T, T^{\prime}\right):=\sup _{v \in T,\|v\|=1} d\left(v, T^{\prime}\right)$ denote the "distance" of vector subspaces of $\mathbb{R}^{n}$. Then $T$ is contained in $T^{\prime}$ if and only if $\delta\left(T, T^{\prime}\right)=0$. It is easy to see that $\delta\left(T, T^{\prime}\right)=\left\|\Pi_{T^{\prime}}^{\perp} \Pi_{T}\right\|$, where $\Pi_{T}$ denotes the projection on $T$ and $\Pi_{T^{\prime}}^{\perp}$ the projection on the orthogonal complement of $T^{\prime}$.

Definition 1.9. A stratification $S=\bigcup_{i} X^{i}$ of a closed subset of $\mathbb{R}^{n}$ is called Verdierstratification if for any pair $X^{i}, X^{j}$ of strata with $X^{i} \subset \partial X^{j}$ and each $x \in X^{i}$ we have the following Verdier condition: There are a real number $C>0$ and a neighborhood $U$ of $x$ such that $\delta\left(T_{p} X^{i}, T_{q} X^{j}\right) \leqslant C\|p-q\|$ for $p \in X^{i} \cap U, q \in X^{j} \cap U$.

Given a Whitney-stratification $S=\cup X^{i} \subset \mathbb{R}^{n}$ of a compact definable set, we denote by $\operatorname{Nor}_{e} S \subset \mathbb{R}^{n} \times S^{n-1} \subset \mathbb{R}^{2 n}$ the set of unit vectors normal to some stratum. Nor $_{e} T_{X^{i}} X^{j}, X^{i} \subset \overline{X^{j}}$ denotes the set of limits of unit normal vectors of $X^{j}$ with footpoints tending to points $X^{i}$. A more detailed description of these sets and their relations can be found in [16].

Definition 1.10. We call a Whitney-stratification $S=\cup X^{i} \subset \mathbb{R}^{n}$ of a compact definable set tame if there is a stratification $\cup N^{\mu}$ of the unit normal space $\operatorname{Nor}_{e} S \subset$ $\mathbb{R}^{n} \times S^{n-1} \subset \mathbb{R}^{2 n}$ satisfying the following two conditions: 
a) $\left\{N^{\mu}\right\}$ is compatible with the sets $\operatorname{Nor}_{e} T_{X^{i}} X^{j}$, where $X^{i} \subset \overline{X^{j}}$. This means that each of the latter sets is a union of strata.

b) The projection $\pi: \operatorname{Nor}_{e} S \rightarrow S,\left(x, v_{e}\right) \mapsto x$ is a submersion on each stratum.

Remark 1.11. Let $S$ be a compact definable set. Then it admits stratifications of each of the above types, as was proven by Ta Lê Loi ([18]) for Verdier stratifications and by Bröcker-Kuppe ([5], [16]) for tame stratifications.

If one defines scalar curvature measure in the integral geometric setting, then one needs tame stratifications from the very beginning. However, in this paper, we define scalar curvature measure by an explicit formula and will need tame stratifications only for some technical arguments in the proof of the main theorem.

We now come to the definition of the scalar curvature measure of definable sets. At first look, this seems to be an ad hoc definition, but it is shown in Theorem 1.2 of [2] that this definition coincides with a more natural definition coming from integral geometry. Since we do not want to go into details on integral geometry (such as Lipschitz-Killing curvatures), we define the scalar curvature measure from the very beginning by the formula below.

Definition 1.12. Let $S$ be a compact connected definable set of dimension $m$ with a stratification $S=\bigcup_{i} X^{i}$. Then we define a (signed) Borel measure by setting for each Borel subset $U \subset S$

$$
\begin{aligned}
\operatorname{scal}(S, U):= & \int_{U \cap X^{m}} s(x) d \operatorname{vol}_{m}(x)+2 \int_{U \cap X^{m-1}} \sum_{i=1}^{k} \operatorname{tr} I I_{w_{i}} d \operatorname{vol}_{m-1}(x) \\
& +4 \pi \int_{U \cap X^{m-2}}\left(\frac{1}{2}+\frac{(-1)^{m}}{2} \chi_{\mathrm{loc}}(S, x)-\theta_{m}(S, x)\right) d \operatorname{vol}_{m-2}(x) .
\end{aligned}
$$

Here, $w_{1}, w_{2}, \ldots, w_{k}$ denote the normal vectors of $X^{m-1}$ in direction of the highest dimensional strata and $\operatorname{tr} I I_{w_{i}}$ is the trace of the second fundamental form of $X^{m-1}$ in direction $w_{i}\left(I I_{w_{i}}=-\nabla w_{i}\right)$. By $\chi_{\mathrm{loc}}(S, x):=\chi\left(H_{*}(S, S \backslash\{x\})\right)$ we denote the local Euler-characteristic of $S$ at $x$ with respect to Borel-Moore homology. $\theta_{m}(S, x)$ denotes the $m$-dimensional density of $S$ at $x$.

Remark 1.13. The scalar curvature measure is independent of the chosen stratification. See [16] or [5] for details.

Examples 1.14. A Riemannian manifold has positive or negative scalar curvature measure if and only if its usual scalar curvature is positive or negative respectively.

A Riemannian manifold with boundary has positive or negative scalar curvature measure if and only if its scalar curvature is positive or negative and the mean curvature of the boundary is positive or negative respectively.

For a compact Riemannian manifold $S, \operatorname{scal}(S, S)$ is called the total scalar curvature. The Einstein-Hilbert functional of $S$ is the functional that associates to a metric the total scalar curvature. 
The scalar curvature measure of the surface of a cube is concentrated in its vertices. The scalar curvature measure of a vertex is $4 \pi\left(1-\frac{3}{4}\right)=\pi$. The total scalar curvature equals $8 \pi=4 \pi \chi$. This is the Gauss-Bonnet-formula, which remains true in this setting. See [5] for details.

\section{Law of reflection}

Not much is known about the behavior of geodesics on definable sets. Approaching the boundary of a highest dimensional stratum, the differential equation for geodesics becomes singular. However, using some metric arguments and sufficiently good stratifications, we are able to show a law of reflection that will be important later on.

Proposition 2.1. Let $S$ be a compact definable set of dimension $m$ with a fixed tame stratification, $X^{m-1}$ and $X^{m}$ strata of dimension $m-1$ respectively $m$ with $X^{m-1} \subset \overline{X^{m}}$. Then there is a definable subset $E$ of $X^{m-1}$ of dimension smaller than $m-1$ such that for each point $P \in X^{m-1} \backslash E$ the following two conditions are satisfied:

- The tangent map extends continuously from $X^{m}$ to $P$, i.e. there is a unique $m$ dimensional space $T$ such that $T_{Q} X^{m} \rightarrow T$ for $Q \rightarrow P$.

- Near P, Verdier's condition is satisfied.

Proof. We can refine the given stratification to a stratification satisfying Verdier's condition. Therefore, if we remove some strata of smaller dimension, Verdier's condition will be satisfied on $X^{m-1}$.

On the other hand, the set of limit tangent spaces of $X^{m}$ has dimension less than $m$ in the Grassmannian of pointed $m-1$ dimensional affine subspaces of $\mathbb{R}^{n}$ (see [16]). We denote for each $x \in \overline{X^{m-1}} \backslash X^{m-1}$ the set of limit tangent spaces at $x$ by $T_{\lim , x} X^{m}$. Then $T_{\lim } X^{m}=\bigcup_{x \in \overline{X^{m}} \backslash X^{m}} T_{\lim , x} X^{m}$. We therefore see that the dimension of $T_{\lim , x} X^{m}$ has to be 0 almost everywhere. As a consequence of Hardt's Theorem (see for instance [10], Theorem 5.22), this dimension is a definable function of $x$. It follows that it is 0 outside a set of smaller dimension. With $X^{m}$ being locally connected, $T_{\lim , P} X^{m}$ is connected for each $P \in X^{m-1}$. Therefore, if it is 0-dimensional, it consists only of a single space which implies that the tangent map extends continuously to $P$.

Proposition 2.2. Let $X^{m}$ and $X^{m-1}$ be two strata as above. Suppose that the tangent map extends continuously from $X^{m}$ to $P$ with limit tangent space $T$. Then we can describe $X^{m}$ (locally at $P$ ) in the following way: There are an orthonormal base of $\mathbb{R}^{n}$ with associated coordinates $\left\{x_{1}, \ldots, x_{n}\right\}$ and smooth functions $g, f$ such that:

$T_{P} X^{m-1}=\left\{x_{m}=x_{m+1}=\cdots=x_{n}=0\right\}$.

$T=\left\{x_{m+1}=x_{m+2}=\cdots=x_{n}=0\right\}$.

$X^{m}$ is the graph of $f$ over the open set $U=\left\{\left(x_{1}, \ldots, x_{m}\right) \in T: x_{m}>g\left(x_{1}, \ldots, x_{m-1}\right)\right\}$.

The derivatives of $f, \frac{\partial f}{\partial x_{i}}(Q)$ tend to 0 for $Q \rightarrow P$. 
Proof. Remember that $X^{m-1}$ is smooth. The tangent space $T_{P} X^{m-1}$ is included in $T$, hence the projection of $X^{m-1}$ to $T$ will be a smooth hypersurface of $T$ which can be written as the graph of a smooth function $g$. If we choose first a coordinate system on $T_{P} X^{m-1}$, extend it to a coordinate system of $T$ and finally to one of $\mathbb{R}^{n}$, we get automatically the first two conditions.

By our assumption, the tangent space $T_{Q} X^{m}$ will be close to $T$ in the Grassmannian. Therefore, the projection from $X^{m}$ to $T$ is a bijective smooth map. By simple topological reasons, its image must be one of the sets $\left\{x_{m}>g\left(x_{1}, \ldots, x_{m-1}\right)\right\}$ or $\left\{x_{m}<g\left(x_{1}, \ldots, x_{m-1}\right)\right\}$. Changing the signs of the coordinate $x_{m}$ and of $g$, we can suppose that this image is $U=\left\{x_{m}>g\left(x_{1}, \ldots, x_{m-1}\right)\right\}$. Since $X^{m}$ is smooth, we get some smooth function $f$ such that $X^{m}$ is the graph of $f$ over $U$. The partial derivatives of $f$ tend to 0 as follows easily from the uniqueness of the limit tangent space.

Remark. We can extend $f$ to a continuous function (also denoted by $f$ ) on $\bar{U}=$ $U \cup \operatorname{graph} g$. The graph of $f$ over the set graph $g$ is nothing else than $X^{m-1}$. Since $X^{m-1}$ is smooth, $\left.f\right|_{\text {graph } g}$ is smooth. We know furthermore that $T_{P} X^{m-1} \subset T$ which yields that all derivatives of $\left.f\right|_{\text {graph } g}$ vanish at 0 .

Proposition 2.3. Same situation as above. Let $A \in X^{m}, P \in X^{m-1}$ be points such that $d_{i}(A, P) \leqslant d_{i}\left(A, P^{\prime}\right)$ for all points $P^{\prime} \in X^{m-1}$. Further suppose that the tangent map extends continuously from $X^{m}$ to $P$. Choose a geodesic $\gamma$ between $P$ and $A$, parameterized by arclength and with $\gamma(0)=P$. Let $w \in T$ be the vector which is given in our coordinate system by $(0, \ldots, 0,1)$. Then

$$
\lim _{\alpha \rightarrow 0} \frac{\gamma(\alpha)-P}{\alpha}=w
$$

Proof. We argue by contradiction. Suppose there exist a vector $w^{\prime} \neq w$ and a sequence of real numbers $\alpha_{1}>\alpha_{2}>\cdots \rightarrow 0$ such that

$$
\lim _{i \rightarrow \infty} \frac{\gamma\left(\alpha_{i}\right)-P}{\alpha_{i}}=w^{\prime}
$$

Set $r_{i}:=\left\|\frac{\gamma\left(\alpha_{i}\right)-P-\alpha_{i} w^{\prime}}{\alpha_{i}}\right\|$. Then $r_{i} \rightarrow 0$. By the triangle inequality we get

$$
r_{i} \alpha_{i} \geqslant d_{e}\left(\gamma\left(\alpha_{i}\right), P\right)-\alpha_{i}\left\|w^{\prime}\right\|
$$

By some easy arguments using Whitney's condition B, we see that $\lim _{i \rightarrow \infty} \frac{d_{e}\left(\gamma\left(\alpha_{i}\right), P\right)}{\alpha_{i}}=$ $\lim _{i \rightarrow \infty} \frac{d_{i}\left(\gamma\left(\alpha_{i}\right), P\right)}{\alpha_{i}}=1$. So we easily get $\left\|w^{\prime}\right\| \geqslant 1$.

On the other hand, we always have $d_{i} \geqslant d_{e}$. It follows $\left\|\gamma\left(\alpha_{i}\right)-P\right\| \leqslant \alpha_{i}$ and

$$
\left\|w^{\prime}\right\| \leqslant\left\|w^{\prime}-\frac{\gamma\left(\alpha_{i}\right)-P}{\alpha_{i}}\right\|+\left\|\frac{\gamma\left(\alpha_{i}\right)-P}{\alpha_{i}}\right\| \leqslant r_{i}+1 .
$$

Since $r_{i} \rightarrow 0$, it follows $\left\|w^{\prime}\right\| \leqslant 1$ and finally $\left\|w^{\prime}\right\|=1$. 
Next, $T$ is the unique limit tangent space and therefore $w^{\prime} \in T$. Since $w^{\prime} \neq w$, some of the first $m-1$ coordinates of $w^{\prime}$ must be non-zero. Suppose without loss of generality that $w^{\prime}=\left(w_{1}, \ldots, w_{m}, 0, \ldots, 0\right)$ with $w_{1} \neq 0$.

Now, $\gamma\left(\alpha_{i}\right)$ has coordinates $\left(h_{1}, \ldots, h_{m}, 0, \ldots, 0\right)$ with $h_{j}=\alpha_{i} w_{j}+o\left(\alpha_{i}\right)$. The line $L_{i}$ that joins this point with the point $P^{\prime}=\left(h_{1}, \ldots, h_{m-1}, g\left(h_{1}, \ldots, h_{m-1}\right), 0, \ldots, 0\right)$ has length $l_{i}=g\left(h_{1}, \ldots, h_{m-1}\right)-h_{m}$. Since the derivatives of $g$ at 0 vanish, it follows that

$$
\lim _{i \rightarrow \infty}\left|\frac{l_{i}}{\alpha_{i}}\right|=\left|w_{m}\right|<1 .
$$

For $i$ sufficiently big, the function $f$ will have arbitrarily small derivatives on $L_{i}$. It follows that the length of the pre-image of $L_{i}$ under the projection to $T$, which is a curve in $X^{m}$, has a length which is close to the length of $L_{i}$, hence strictly smaller than $\alpha_{i}$. This is a contradiction, since we have supposed that there is no point on $X^{m-1}$ with smaller distance to $A$ than $P$, but $P^{\prime}$ would be such a point.

A similar proof will yield the following:

Proposition 2.4 (Law of reflection). Let $S$ be a compact connected definable set of dimension $m$ with a fixed tame stratification and $X^{m-1}$ an $m-1$ stratum neighboring exactly two m-strata $X_{1}^{m}, X_{2}^{m}$. Let $\gamma$ be a geodesic such that $\gamma(\alpha) \in X_{1}^{m}$ for $\alpha<0, P=$ $\gamma(0) \in X^{m-1}$ and such that $\gamma$ can be extended beyond $P$. We assume that the tangent map extends continuously from $X_{1}^{m}, X_{2}^{m}$ to $P$. We choose for each stratum $X_{i}^{m}$ a representation as in Proposition 2.2. Since both $T_{1}$ and $T_{2}$ contain $T_{P} X^{m-1}$, we can suppose that the coordinates $x_{1}, \ldots, x_{m-1}$ coincide on $T_{P} X^{m-1}$. Let $w_{1}=\left(a_{1}, \ldots, a_{m}, 0, \ldots, 0\right)_{1}$ be a tangent vector of $\left.\gamma\right|_{<0}$. Then, if $w_{1}$ is not contained in $T_{P} X^{m-1},\left.\gamma\right|_{>0}$ has a unique tangent vector $w_{2}$ at $P$ which is given by $w_{2}=\left(-a_{1}, \ldots,-a_{m-1}, a_{m}, 0, \ldots, 0\right)_{2}$.

Remarks. a) If $w_{1}$ is contained in $T_{P} X^{m-1}$, the same holds true for $w_{2}$, for otherwise we could apply the proposition with the reversed geodesic.

b) If $w_{1}$ is not contained in $T_{P} X^{m-1}$, both sides of $\gamma$ have unique tangent vectors. (Just apply the proposition twice.) It follows that $\gamma(\alpha)=P+\alpha w_{1}+o(\alpha)$ and $\gamma(-\alpha)=$ $P+\alpha w_{2}+o(\alpha)$ for small positive $\alpha$.

Proof of the proposition. Assume first that there is a sequence $\alpha_{1}>\alpha_{2}>\cdots \rightarrow 0$ such that $\gamma\left(\alpha_{i}\right) \in \bar{X}_{1}^{m}$ for each $i$. Then the set

$$
\left\{\frac{\gamma\left(\alpha_{i}\right)-P}{\alpha_{i}} \mid i=1,2, \ldots\right\}
$$

has an accumulation point $w^{\prime}$ of norm 1 (this follows from arguments as in Proposition 2.3). By choosing a subsequence of $\left\{\alpha_{i}\right\}$ (which for simplicity we call again $\alpha$ ) we can thus suppose that

$$
\gamma\left(\alpha_{i}\right)=P+\alpha_{i} w^{\prime}+o\left(\alpha_{i}\right) .
$$

Let $w^{\prime}$ be given in coordinates by $w^{\prime}=\left(a_{1}^{\prime}, a_{2}^{\prime}, \ldots, a_{m}^{\prime}, 0, \ldots, 0\right)$. Let $\Pi_{1}$ be the projection of $\mathbb{R}^{n}$ to $T_{1}$. Since all derivatives of $g$ at 0 vanish, the line between the points 
$\Pi_{1} \gamma\left(\alpha_{i}\right)$ and $\Pi_{1} \gamma\left(-\alpha_{i}\right)$ lies entirely in $U$ for $\alpha_{i}$ small enough. Its length is given by $\alpha_{i}\left\|w^{\prime}-w\right\|+o\left(\alpha_{i}\right)$. The pre-image of this line yields a curve between $\gamma\left(\alpha_{i}\right)$ and $\gamma\left(-\alpha_{i}\right)$ of length bounded by

$$
(1+o(1))\left(\alpha_{i}\left\|w^{\prime}-w\right\|+o\left(\alpha_{i}\right)\right)=\alpha_{i}\left\|w^{\prime}-w\right\|+o\left(\alpha_{i}\right)
$$

This follows from the fact that the derivatives of $f$ tend to 0 for $\alpha_{i} \rightarrow 0$.

On the other hand, $\gamma$ is a geodesic between $\gamma\left(\alpha_{i}\right)$ and $\gamma\left(-\alpha_{i}\right)$ which shows

$$
d_{i}\left(\gamma\left(\alpha_{i}\right), \gamma\left(-\alpha_{i}\right)\right)=2 \alpha_{i}
$$

From both inequalities we easily deduce $\left\|w^{\prime}-w\right\| \geqslant 2$. This is a contradiction, since $w^{\prime}$ and $w$ both have norm 1 and have coordinates $a_{m}>0$ respectively $a_{m}^{\prime} \geqslant 0$. Our assumption was wrong, therefore $\gamma(\alpha) \in X_{2}^{m}$ for $\alpha$ in an interval $(0, \varepsilon), \varepsilon>0$.

Take $w^{\prime \prime}$ and a sequence $\alpha_{1}>\alpha_{2}>\cdots \rightarrow 0$ with

$$
\gamma\left(\alpha_{i}\right)=P+\alpha_{i} w^{\prime \prime}+o\left(\alpha_{i}\right)
$$

We shall show that $w^{\prime \prime}=w_{2}$.

Again, $\left\|w^{\prime \prime}\right\|=1$ by easy arguments.

Consider in $\mathbb{R}^{m}$ the points $\left(a_{1}, \ldots, a_{m}\right)$ and $\left(a_{1}^{\prime}, \ldots, a_{m-1}^{\prime},-a_{m}^{\prime}\right)$. Since $a_{m}>0$ and $a_{m}^{\prime} \geqslant 0$, the line between these points intersects the set $\left\{x_{m}=0\right\}$ in a point $b=$ $\left(b_{1}, \ldots, b_{m-1}, 0\right)$.

For $\alpha_{i}$ small, the line in $T_{1}$ between $\Pi_{1} \gamma\left(-\alpha_{i}\right)$ and $\left(b, g_{1}(b), 0, \ldots, 0\right)_{1}$ lies in $U_{1}$ and has a certain length $l_{1}$, while the line between $\Pi_{2} \gamma\left(\alpha_{i}\right)$ and $\left(b, g_{2}(b), 0, \ldots, 0\right)_{2}$ lies in $U_{2}$ and has a certain length $l_{2}$. From the fact that $g$ is smooth with vanishing derivatives at 0 , we deduce that

$$
l_{1}+l_{2}=\alpha_{i}\left\|\left(a_{1}-a_{1}^{\prime}, \ldots, a_{m-1}-a_{m-1}^{\prime}, a_{m}+a_{m}^{\prime}\right)\right\|+o\left(\alpha_{i}\right) .
$$

Again, we can lift the union of these lines by $\Pi_{1}$ and $\Pi_{2}$ to get a curve joining $\gamma\left(\alpha_{i}\right)$ and $\gamma\left(-\alpha_{i}\right)$ whose length is bounded by

$$
\alpha_{i}\left\|\left(a_{1}-a_{1}^{\prime}, \ldots, a_{m-1}-a_{m-1}^{\prime}, a_{m}+a_{m}^{\prime}\right)\right\|+o\left(\alpha_{i}\right) .
$$

This cannot be smaller than $d_{i}\left(\gamma\left(\alpha_{i}\right), \gamma\left(-\alpha_{i}\right)\right)=2 \alpha_{i}$ since $\gamma$ is a geodesic between both points. We deduce that

$$
\left\|\left(a_{1}-a_{1}^{\prime}, \ldots, a_{m-1}-a_{m-1}^{\prime}, a_{m}+a_{m}^{\prime}\right)\right\| \geqslant 2 .
$$

Finally, it follows that $a_{1}^{\prime}=-a_{1}, a_{2}^{\prime}=-a_{2}, \ldots, a_{m-1}^{\prime}=-a_{m-1}, a_{m}^{\prime}=a_{m}$.

Proposition 2.5. Let $S$ be a compact definable set of dimension $m$ with a fixed tame stratification. Suppose $S$ has the geodesic extension property and $X^{m-1}$ is a stratum neighboring exactly two strata $X_{1}^{m}, X_{2}^{m}$ of highest dimension. Then at a dense set of points $P \in X^{m-1}$ there is a geodesic $\gamma$ passing through both sides of $X^{m-1}$ that has unique tangent vectors at $P$ which are furthermore orthogonal to $T_{P} X^{m-1}$. 
Proof. Choose some point $A \in X_{1}^{m}$ near $X^{m-1}$ and the point $P$ which is the nearest to $A$ in the inner metric. It is clear that there is a dense subset of points $P$ arising in this way. So we can furthermore assume that $P$ satisfies both conditions of Proposition 2.1 and we can apply Propositions 2.4 and 2.3.

Choose a geodesic between $A$ and $P$ and extend it beyond $P$ to a geodesic $\gamma$. We can reparameterize by arclength such as to have $\gamma(0)=P, \gamma\left(-d_{i}(A, P)\right)=A$. By Proposition 2.4, $\gamma(\alpha) \in X_{2}^{m}$ for small positive $\alpha$. We know by Proposition 2.3 that $\left.\gamma\right|_{<0}$ has a unique tangent vector $w_{1}=(0, \ldots, 0,1)_{1}$ (the index refers to the coordinate system). We can therefore apply Proposition 2.4 to see that $\gamma_{>0}$ must have $w_{2}=(0, \ldots, 0,1)_{2}$ as unique tangent vector. This finishes the proof of the proposition.

\section{Proof of the Main Theorem}

We recall that the Main Theorem states that a compact connected definable set of dimension $m$ which is a pseudo-manifold with curvature bounded from above by $\kappa$ has scalar curvature bounded from above by $\kappa m(m-1)$, which means that $\operatorname{scal}(S,-) \leqslant \kappa m(m-1) \operatorname{vol}(S,-)$. Before turning to the proof, we will give two easy examples.

Example 3.1. Let $S$ be a connected two-dimensional piecewise linear space. Suppose that $S$ is a topological manifold. Then the following statements are equivalent:

a) $S$ is a metric space of curvature $\leqslant 0$.

b) For every vertex $v$ of $S$, the link of $S$ at $v$ is a CAT(1)-space.

c) The density at each vertex is at least 1 .

d) $\operatorname{scal}(S,-) \leqslant 0$.

The equivalence between a) and b) is remarked in [4], Theorem 5.2., the equivalence between $b$ ) and c) comes from the fact that a one-dimensional space is CAT(1) if and only if there are no loops of length smaller than $2 \pi$. The equivalence between $\mathrm{c}$ ) and d) is clear by definition.

Example 3.2. Let us generalize this example to the higher dimensional case. $S$ is now a piecewise linear manifold which is supposed to be a topological manifold and to have curvature bounded from above by 0 .

Let $m$ denote the dimension of $S$. The scalar curvature measure is concentrated on simplices of dimension $m-2$. Locally at a point $P$ of such a simplex, $S$ is the product of the normal section and $\mathbb{R}^{m-2}$. Consequently, the normal section is (at least locally) a convex subset of $S$ and thus a CAT(0)-space in a neighborhood of $P$ (see also Example II 1.15 of [4]). Since it is a two-dimensional space, this is by the preceding example equivalent to the density at $P$ being at least 1 , which implies non-positive scalar curvature measure by Definition 1.12.

Proof of Theorem 1.1. Let $S$ be a compact connected definable pseudo-manifold of dimension $m$ which has curvature bounded from above by $\kappa$. Choose a tame stratification $S=\cup X^{i}$ of $S$. By the topological condition, refining this stratification if nec- 
essary, we can assume that each stratum of codimension 1 lies in the boundary of exactly two $m$-strata, hence in Formula 1.12 we have $k=2$.

Furthermore, by Thom's Isotopy Lemma ([20], [14]), we get for each point $x \in X^{m-2}$ that $\chi_{\text {loc }}(S, x)=(-1)^{m}$. In view of Definition 1.12, we have to show that for each Borel subset $U \subset S$, the expression

$$
\begin{aligned}
\operatorname{scal}(S, U)= & \int_{U \cap X^{m}} s(x) d \operatorname{vol}_{m}(x)+2 \int_{U \cap X^{m-1}} \operatorname{tr} I I_{w_{1}+w_{2}} d \operatorname{vol}_{m-1}(x) \\
& +4 \pi \int_{U \cap X^{m-2}}\left(1-\theta_{m}(S, x)\right) d \operatorname{vol}_{m-2}(x)
\end{aligned}
$$

is bounded from above by $\kappa m(m-1) \operatorname{vol}(U)$.

This can be done for each stratum dimension separately, the codimension 0 case being trivial (the CAT $(\kappa)$-condition implies $s(x) \leqslant \kappa m(m-1)$ on the smooth part). For strata of codimension 1 and 2, we will show the non-positivity of the scalar curvature measure in Propositions 3.3 and 3.6.

3.1 Strata of codimension 1. The aim of this section is the proof of the following:

Proposition 3.3. If $S$ is a compact connected definable set of dimension $m$ which is a $\mathrm{CAT}(\kappa)$-space for some $\kappa$ (with respect to the inner metric) and $X^{m-1}$ an $m-1$-stratum on the boundary of exactly two m-strata, then $\left.\operatorname{scal}(S,-)\right|_{X^{m-1}} \leqslant 0$

We recall that $\left.\operatorname{scal}(S,-)\right|_{X^{m-1}}$ is given by integration of the definable function $h:=$ $2 \operatorname{tr} I I_{w_{1}+w_{2}}$ on $X^{m-1}$. To show that $\left.\operatorname{scal}(S,-)\right|_{X^{m-1}} \leqslant 0$, it is therefore enough to establish that $h(P) \leqslant 0$ on a dense subset of $X^{m-1}$. We are going to prove that $h(P) \leqslant 0$ for all points $P \in X^{m-1}$ such that:

a) The tangent map can be extended continuously from both $X_{1}^{m}, X_{2}^{m}$ to $P$.

b) The stratification satisfies Verdier's condition near $P$.

c) There is a geodesic passing through $P$ as in Proposition 2.5.

Let $S$ have curvature $\leqslant \kappa$ and let $S=\cup X^{i}$ be a tame stratification of $S$ and $X^{m-1}$ a stratum neighboring exactly two $m$-strata. Then by Propositions 2.1 and 2.5 , the set of points $P \in X^{m-1}$ satisfying the above three conditions is a dense subset of $X^{m-1}$.

Let us fix such a point $P$. We find a geodesic $\gamma$ consisting of two geodesic arcs $\gamma_{1}, \gamma_{2}$ such that $\gamma_{i}$ lies entirely in $X_{i}^{m}$ and such that

$$
\gamma_{i}(\alpha)=P+\alpha w_{i}+r_{i}(\alpha)
$$

for small positive $\alpha$ and where $r_{i}$ denotes a function with $\lim _{\alpha \rightarrow 0} \frac{r_{i}(\alpha)}{\alpha}=0$. Furthermore, $w_{i} \perp T_{P} X^{m-1}$ and $\left\|w_{i}\right\|=1$.

Choose real functions $\varepsilon_{1}, \varepsilon_{2}:(0, \infty) \rightarrow(0, \infty)$ such that for $i=1,2$

a) $\varepsilon_{i}$ is monotonically increasing,

b) $\lim _{\alpha \rightarrow 0} \varepsilon_{i}(\alpha)=0$,

c) $\left\|\frac{r_{i}(\alpha)}{\alpha}\right\| \leqslant \varepsilon_{i}(\alpha)$. 
We carry out the following construction for both $X_{1}^{m}$ and $X_{2}^{m}$. In order to simplify the notation, we omit indices. We write $\gamma$ in place of $\gamma_{i}$ and so on. The well defined limit tangent space of $X^{m}$ at $P$ is denoted by $T$.

Apply Proposition 2.2 to the stratum $X^{m}$ and the point $P$. This yields a system of coordinates $x_{1}, \ldots, x_{n}$, a set

$$
U=\left\{\left(x_{1}, \ldots, x_{m}\right) \in \mathbb{R}^{m}: x_{m}>g\left(x_{1}, \ldots, x_{m-1}\right)\right\} \subset T=\left\{x_{m-1}=\cdots=x_{n}=0\right\}
$$

and a function $f: U \rightarrow \mathbb{R}^{n-m}$ such that $X^{m}$ is the graph of $f$ and $f$ has derivatives that converge to 0 if we approach $P$. In coordinates, $f$ can be written as $f=$ $\left(f_{1}, \ldots, f_{n-m}\right)$. Since $P$ is contained in $T$, we get $f(0, \ldots, 0)=0$.

Lemma 3.4. There are a neighborhood $V_{P}$ of $P$ and a constant $C>0$ such that the function $f$ satisfies for each $j=1, \ldots, n-m$ the following estimate:

$$
\left|\frac{\partial f_{j}}{\partial x_{i}}(Q)\right| \leqslant C d_{e}(Q, P) \quad \text { for } i=1, \ldots, m-1 \text { and } Q \in V_{P} .
$$

Furthermore,

$$
\left|\frac{\partial f_{j}}{\partial x_{m}}(Q)\right| \rightarrow 0 \quad \text { for } Q \rightarrow P .
$$

Proof of the lemma. The second assertion follows from the fact that the tangent map can be extended continuously from $X^{m}$ to $P$ (see Proposition 2.2).

Let us sketch the proof of the first assertion. It will be a consequence of Verdier's condition. Remark that the tangent space of $X^{m}$ at $Q$ is generated as a vector space by the vectors

$$
\left(1,0, \ldots, 0, \frac{\partial f_{1}}{\partial x_{1}}, \ldots, \frac{\partial f_{n-m}}{x_{1}}\right), \ldots,\left(0, \ldots, 0,1, \frac{\partial f_{1}}{\partial x_{m}}, \ldots, \frac{\partial f_{n-m}}{x_{m}}\right) .
$$

Since the tangent spaces $T_{Q} X^{m}$ converge to $T$ for $Q \rightarrow P$, we see that all derivatives converge to 0 . We can suppose without loss of generality that among the vectors $\frac{\partial f}{\partial x_{i}}(Q), i=1, \ldots, m-1$, the first one is the longest. We denote its length by $L=L(Q)$. We have to show that $L(Q) \leqslant C d_{e}(P, Q)$ for some constant $C>0$.

Let $\Pi_{P}$ denote the orthogonal projection to $T_{P} X^{m-1}$ and $\Pi_{Q}^{\perp}$ the orthogonal projection to the orthogonal complement of $T_{Q} X^{m}$. From Verdier's condition we know that $\left\|\Pi_{Q}^{\perp} \Pi_{P}\right\| \leqslant C d_{e}(P, Q)$ for some constant $C>0$.

Consider the unit vector $(1,0, \ldots, 0) \in T_{P} X^{m-1}$. Its projection $\Pi_{Q}(1,0, \ldots, 0)$ to $T_{Q} X^{m}$ is given by

$$
\left(a_{1}, a_{2}, \ldots, a_{m}, a_{1} \frac{\partial f_{1}}{\partial x_{1}}+\cdots+a_{m} \frac{\partial f_{1}}{\partial x_{m}}, \ldots, a_{1} \frac{\partial f_{n-m}}{\partial x_{1}}+\cdots+a_{m} \frac{\partial f_{n-m}}{\partial x_{m}}\right)
$$

for some real numbers $\left(a_{1}, \ldots, a_{m}\right)$. The condition of orthogonality yields a system of linear equations for the unknowns $a_{1}, \ldots, a_{m}$. It has the form 


$$
(\operatorname{Id}+o(L))\left(a_{1}, \ldots, a_{m}\right)^{T}=(1,0, \ldots, 0)^{T}
$$

where $\operatorname{Id}_{m}$ denotes the identity matrix of type $(m, m)$ and $o(L)$ is a matrix that tends faster to 0 than $L$.

Resolution gives us $a_{1}=1+o(L), a_{2}=o(L), \ldots, a_{m}=o(L)$ where $o(L)$ stands for real numbers that tend faster to 0 than $L$. Hence

$$
\Pi_{Q}^{\perp} \Pi_{P}(1,0, \ldots, 0)=\left(o(L), o(L), \ldots, o(L), \frac{\partial f_{1}}{\partial x_{1}}+o(L), \ldots, \frac{\partial f_{n-m}}{\partial x_{1}}+o(L)\right)
$$

and therefore

$$
\left\|\Pi_{Q}^{\perp} \Pi_{P}(1,0, \ldots, 0)\right\|=\left\|\left(0, \ldots, 0, \frac{\partial f_{1}}{\partial x_{1}}, \ldots, \frac{\partial f_{n-m}}{\partial x_{1}}\right)\right\|+o(L)=L+o(L) .
$$

We know from Verdier's condition that this must be bounded by $C d_{e}(P, Q)$ for some constant $C>0$. Since we already know that $L$ tends to 0 for $Q \rightarrow P$, we see that $L$ is bounded by $2 C d_{e}(P, Q)$ for $Q$ sufficiently near $P$. This proves the lemma.

Next, choose some real function $\varepsilon_{3}:(0, \infty) \rightarrow(0, \infty)$ such that:

a) $\varepsilon_{3}$ is monotonically increasing,

b) $\lim _{s \rightarrow 0} \varepsilon_{3}(s)=0$,

c) for all $s>0, Q \in B_{e}(P, s)$ and $j=1, \ldots, n-m$ we have $\left|\frac{\partial f_{j}}{\partial x_{m}}(Q)\right| \leqslant \varepsilon_{3}(s)$.

Remember that there are two $m$-strata neighboring $X^{m-1}$. We define $\varepsilon_{4}$ in the same way as we did for $\varepsilon_{3}$, but this time for the other $m$-strata. The existence of such functions follows from the second assertion of the lemma.

We denote by $\Pi_{T}$ the orthogonal projection from $S$ to $T$.

Let $s$ be a positive real number which we will let tend to 0 . Set

$$
\begin{gathered}
\varepsilon(s):=\max \left\{s, \varepsilon_{1}(s), \varepsilon_{2}(s), \varepsilon_{3}(s), \varepsilon_{4}(s)\right\}^{1 / 2}, \\
\alpha(s):=s^{2} / \varepsilon(s), \\
A=A(s):=x(s) \in X^{m-1}, \quad \bar{A}:=\Pi_{T} A \in \operatorname{graph} g \subset \bar{U} \subset T, \\
B=B(\alpha):=\gamma(\alpha), \quad \bar{B}:=\Pi_{T}(B) \in U \subset T .
\end{gathered}
$$

Note that $\varepsilon$ is monotonically increasing and $\lim _{s \rightarrow 0} \varepsilon(s)=0$. We easily see that $\alpha \leqslant s$ for $s \leqslant 1$.

Remember that $\gamma(\alpha)=P+\alpha w+r(\alpha)$ where the vector $w \in T$ is given in our coordinate system by $(0, \ldots, 0,1)$ and where $r(\alpha)=o(\alpha)$. Then $\bar{B}$ is given by $(0, \ldots, 0, \alpha)+\bar{r}(\alpha)$. Hence, $\frac{\|\bar{r}(\alpha)\|}{\alpha}$ tends to 0 for $\alpha \rightarrow 0$.

Since $x_{1}(s)$ is a geodesic on $X^{m-1}, x_{1}^{\prime \prime}(0)$ is orthogonal to $T_{P} X^{m-1}$. From $x_{1}(s)=$ $P+s x_{1}^{\prime}(0)+\frac{s^{2}}{2} x_{1}^{\prime \prime}(0)+O\left(s^{3}\right)$ we get

$$
\bar{A}(s)=\left(s, 0, \ldots, 0, \frac{s^{2}}{2}\left\langle x_{1}^{\prime \prime}(0), w\right\rangle\right)+O\left(s^{3}\right) .
$$


Lemma 3.5. For sufficiently small s, the line between $\bar{A}$ and $\bar{B}$ lies entirely in $U$.

Remark. Here is where we need that $\alpha$ is strictly bigger than $s^{2}$. On the other hand, we will need later that the quotient $\frac{s^{2}}{\alpha}$ should very slowly tend to 0 in order to erase some superfluous terms. This is why we defined $\alpha$ in such a complicated way. Compare also with the situation for Alexandrov spaces with curvature bounded from below ([2]), where a similar definition for $\alpha$ was necessary.

Proof. Suppose there are arbitrarily small positive $s$ such that the line does not lie entirely in $U$. The slope of this line in direction $x_{m}$ is of order $\alpha / s=s / \varepsilon(s)$. If the line does not lie in $U$, it must cut the graph of $g$ at some point between $\bar{A}$ and $\bar{B}$. Then, at some other point, the slope of the graph of $g$ would equal the slope of the line.

Remember that $g$ is smooth with vanishing derivatives at 0 . Therefore the derivatives of $g$ behave like $O(s)$. This is a contradiction, since $\varepsilon(s)$ tends to 0 for $s \rightarrow 0$. This shows the lemma.

Let $\bar{L}$ denote the line between $\bar{A}$ and $\bar{B}$. It is given by

$$
\begin{aligned}
\bar{L}(t)=(1-t) \bar{A}+t \bar{B}= & \left((1-t) s, 0, \ldots, 0, t \alpha+(1-t) \frac{s^{2}}{2}\left\langle x_{1}^{\prime \prime}(0), w\right\rangle\right) \\
& +t \bar{r}(\alpha)+O\left(s^{3}\right) \quad 0 \leqslant t \leqslant 1 .
\end{aligned}
$$

Let $L$ denote the pre-image of $\bar{L}$ under $\Pi$. Since $\bar{L}$ lies in $U, L$ is well defined and yields a curve in $S$ between $A$ and $B$. In our coordinate system, $L$ is given by

$$
L(t)=\left(\bar{L}(t), f_{1}(\bar{L}), f_{2}(\bar{L}), \ldots, f_{n-m}(\bar{L})\right) .
$$

We will compute its length in order to get an upper bound for $d_{i}(A, B)$. First, note the following estimates:

$$
\begin{gathered}
\left|\bar{B}_{1}-\bar{A}_{1}\right|=\left|-s+\bar{r}_{1}(\alpha)\right| \leqslant O(s), \\
\left|\bar{B}_{k}-\bar{A}_{k}\right|=\left|\bar{r}_{k}(\alpha)\right| \leqslant O(\alpha) \leqslant O(s), \quad k=2, \ldots, m-1, \\
\left|\bar{B}_{m}-\bar{A}_{m}\right|=\left|-\frac{s^{2}}{2}\left\langle x_{1}^{\prime \prime}(0), w\right\rangle+\alpha+\bar{r}_{m}(\alpha)+O\left(s^{3}\right)\right| \leqslant O(\alpha) \leqslant O(s) .
\end{gathered}
$$

On the other hand, since the first component of $\bar{B}-\bar{A}$ is $-s+\bar{r}_{1}(\alpha)$, we have $\|\bar{B}-\bar{A}\| \geqslant C_{2} s$ for some constant $C_{2}$.

$$
L^{\prime}(t)=\left(\bar{L}^{\prime}(t), \frac{\partial}{\partial t} f_{1}(\bar{L}), \frac{\partial}{\partial t} f_{2}(\bar{L}), \ldots, \frac{\partial}{\partial t} f_{n-m}(\bar{L})\right) .
$$

Obviously, $\bar{L}^{\prime}(t)=\bar{B}-\bar{A}$. We calculate the other derivatives. It suffices to do the calculation for $\frac{\partial}{\partial t} f_{1}(\bar{L})$ : 


$$
\frac{\partial}{\partial t} f_{1}(\bar{L})=\sum_{k=1}^{m} \frac{\partial f}{\partial x_{k}} \frac{\partial \bar{L}_{k}}{\partial t}
$$

We claim that this is an $O\left(s^{2}\right)$-term. For $k=1,2, \ldots, m-1$, we have $\left|\frac{\partial f}{\partial x_{k}}\right| \leqslant O(s)$ and $\left|\frac{\partial \bar{L}_{k}}{\partial t}\right| \leqslant O(s)$. For $k=m$ and small $s$ we have $\left|\frac{\partial f}{\partial x_{k}}\right| \leqslant \varepsilon_{3}(s) \leqslant \varepsilon(s)$ and $\left|\frac{\partial \bar{L}_{k}}{\partial t}\right| \leqslant$ $O(\alpha)$. The product is $\leqslant O(\varepsilon(s) \alpha)=O\left(s^{2}\right)$. This shows the claim.

It follows that

$$
\left\|L^{\prime}(t)\right\|^{2} \leqslant\left\|\bar{L}^{\prime}(t)\right\|^{2}+O\left(s^{4}\right)=\|\bar{B}-\bar{A}\|^{2}+O\left(s^{4}\right) \leqslant\left(1+O\left(s^{2}\right)\right)\|\bar{B}-\bar{A}\|^{2} .
$$

From this we can deduce

$$
d_{i}(A, B) \leqslant l(L)=\int_{0}^{1}\left\|L^{\prime}(t)\right\| d t \leqslant\left(1+O\left(s^{2}\right)\right)\|\bar{B}-\bar{A}\| .
$$

The inequality $d_{i}(A, B) \geqslant d_{e}(A, B) \geqslant d_{e}(\bar{A}, \bar{B})=\|\bar{B}-\bar{A}\|$ implies that in fact

$$
d_{i}(A, B)=\left(1+O\left(s^{2}\right)\right)\|\bar{B}-\bar{A}\| \text {. }
$$

In our coordinate system, $\bar{B}-\bar{A}$ is represented as

$$
\bar{B}-\bar{A}=\left(-s+\bar{r}_{1}(\alpha), \bar{r}_{2}(\alpha), \ldots, \bar{r}_{m-1}(\alpha), \alpha+\bar{r}_{m}(\alpha)-\frac{s^{2}}{2}\left\langle x_{1}^{\prime \prime}(0), w\right\rangle\right)+O\left(s^{3}\right) .
$$

It follows that

$$
\begin{aligned}
\|\bar{B}-\bar{A}\|^{2}= & s^{2}-2 s \bar{r}_{1}(\alpha)+O\left(\bar{r}_{1}(\alpha)^{2}\right)+\cdots+O\left(\bar{r}_{m}(\alpha)^{2}\right) \\
& +\alpha^{2}+O\left(\alpha \bar{r}_{m}(\alpha)\right)-\alpha s^{2}\left\langle x_{1}^{\prime \prime}(0), w\right\rangle+O\left(\bar{r}_{m}(\alpha) s^{2}\right)+O\left(s^{4}\right) \\
= & s^{2}-2 s \bar{r}_{1}(\alpha)+\alpha^{2}-\alpha s^{2}\left\langle x_{1}^{\prime \prime}(0), w\right\rangle \\
& +O\left(\left(\alpha \varepsilon_{1}(\alpha)\right)^{2}\right)+O\left(\alpha^{2} \varepsilon_{1}(\alpha)\right)+O\left(\alpha \varepsilon_{1}(\alpha) s^{2}\right)+O\left(s^{4}\right) .
\end{aligned}
$$

Next we have for $s \rightarrow 0$ :

$$
\begin{aligned}
\frac{\alpha^{2} \varepsilon_{1}(\alpha)^{2}}{\alpha s^{2}} & \leqslant \frac{\alpha \varepsilon_{1}(s)^{2}}{s^{2}} \leqslant \frac{\alpha \varepsilon(s)^{4}}{s^{2}}=\varepsilon(s)^{3} \rightarrow 0, \\
\frac{\alpha^{2} \varepsilon_{1}(\alpha)}{\alpha s^{2}} & \leqslant \frac{\alpha \varepsilon_{1}(s)}{s^{2}} \leqslant \frac{\alpha \varepsilon(s)^{2}}{s^{2}}=\varepsilon(s) \rightarrow 0, \\
\frac{\alpha \varepsilon_{1}(\alpha) s^{2}}{\alpha s^{2}} & =\varepsilon_{1}(\alpha) \rightarrow 0, \\
\frac{s^{4}}{\alpha s^{2}} & =\varepsilon(s) \rightarrow 0 .
\end{aligned}
$$

It follows that

$$
\|\bar{B}-\bar{A}\|^{2}=s^{2}-2 s \bar{r}_{1}(\alpha)+\alpha^{2}-s^{2}\left\langle x_{1}^{\prime \prime}(0), w\right\rangle+o\left(\alpha s^{2}\right) .
$$


From simple calculations and the fact that $\gamma$ is a geodesic between $P$ and $B$ we see that

$$
s \geqslant d_{i}(P, A) \geqslant d_{e}(P, A)=s+O\left(s^{3}\right)
$$

and

$$
d_{i}(P, B)=\alpha
$$

This enables us to compute the angle $\angle(A, P, B)$ in the case $\kappa=0$ :

$$
\begin{aligned}
\cos \angle & (A, P, B) \\
& =\frac{d(P, A)^{2}+d(P, B)^{2}-d(A, B)^{2}}{2 d(P, A) d(P, B)} \\
& =\frac{s^{2}+\alpha^{2}-\left(1+O\left(s^{2}\right)\right)\left(s^{2}-2 s \bar{r}_{1}(\alpha)+\alpha^{2}-\alpha s^{2}\left\langle x_{1}^{\prime \prime}(0), w\right\rangle+o\left(\alpha s^{2}\right)\right)+O\left(s^{4}\right)}{2 \alpha\left(s+O\left(s^{2}\right)\right)} \\
& =\frac{\bar{r}_{1}(\alpha)}{\alpha}+\frac{s}{2}\left\langle x_{1}^{\prime \prime}(0), w\right\rangle+o(s) .
\end{aligned}
$$

(Remark. In the case $\kappa \neq 0$, we have to apply the corresponding law of cosines, but the resulting asymptotic behavior is the same. This is not surprising, as we consider very small triangles and locally, differences between hyperbolic, spherical and Euclidean space vanish.)

Doing the same calculations with $A=x_{1}(s)$ replaced by $C=x_{1}(-s)$ yields

$$
\cos \angle(C, P, B)=\frac{-\bar{r}_{1}(\alpha)}{\alpha}+\frac{s}{2}\left\langle x_{1}^{\prime \prime}(0), w\right\rangle+o(s) .
$$

Furthermore, replacing $B=\gamma_{1}\left(\alpha_{1}\right)$ by $D=\gamma_{2}\left(\alpha_{2}\right)$ gives us:

$$
\begin{aligned}
& \cos \angle(A, P, D)=\frac{\bar{r}_{2}\left(\alpha_{2}\right)}{\alpha_{2}}+\frac{s}{2}\left\langle x_{1}^{\prime \prime}(0), w_{2}\right\rangle+o(s), \\
& \cos \angle(C, P, D)=\frac{-\bar{r}_{2}\left(\alpha_{2}\right)}{\alpha_{2}}+\frac{s}{2}\left\langle x_{1}^{\prime \prime}(0), w_{2}\right\rangle+o(s) .
\end{aligned}
$$

Taking the sum of the cosines of these four angles yields:

$$
\begin{aligned}
\cos \angle & (A, P, B)+\cos \angle(C, P, B)+\cos \angle(A, P, D)+\cos \angle(C, P, D) \\
& =s\left\langle x_{1}^{\prime \prime}(0), w_{1}+w_{2}\right\rangle+o(s)
\end{aligned}
$$

Let $\gamma_{A}, \gamma_{B}=\gamma_{1}, \gamma_{C}, \gamma_{D}=\gamma_{2}$ denote the geodesics between $P$ and $A, B, C, D$. By the CAT-inequality, we have

$$
\angle P\left(\gamma_{A}, \gamma_{B}\right) \leqslant \angle(A, P, B)
$$


and analogous inequalities for the other points. From the triangle inequality for angles (see [4], Proposition 1.14) and the fact that $B, P, D$ lie on a geodesic $\gamma$, we get

$$
\pi=\angle P\left(\gamma_{B}, \gamma_{D}\right) \leqslant \angle P\left(\gamma_{B}, \gamma_{A}\right)+\angle{ }_{P}\left(\gamma_{A}, \gamma_{D}\right) \leqslant \angle(A, P, B)+\angle(A, P, D)
$$

Using monotony of the cosine function, we get

$$
\cos \angle(A, P, B)+\cos \angle(A, P, D) \leqslant 0
$$

and analogously

$$
\cos \angle(C, P, B)+\cos \angle(C, P, D) \leqslant 0 \text {. }
$$

It follows that

$$
s\left\langle x_{1}^{\prime \prime}(0), w_{1}+w_{2}\right\rangle+o(s) \leqslant 0
$$

and hence

$$
\left\langle x_{1}^{\prime \prime}(0), w_{1}+w_{2}\right\rangle \leqslant 0 .
$$

In the same way, such an inequality holds true with $x_{1}$ replaced by $x_{i}$ for $i=$ $1 \ldots, m-1$. This shows

$$
h(P)=2\left(\operatorname{tr} I I_{w_{1}}+\operatorname{tr} I I_{w_{2}}\right)=2 \sum_{j=1}^{m-1}\left\langle x_{j}^{\prime \prime}(0), w_{1}+w_{2}\right\rangle \leqslant 0
$$

which was to be shown.

\subsection{Strata of codimension 2 .}

Proposition 3.6. Let $S$ be a compact connected definable set of dimension $m$ with a fixed tame stratification. Suppose that $S$ is a space of curvature $\leqslant \kappa$ for some $\kappa \in \mathbb{R}$ and that each $m-1$-stratum lies on the boundary of exactly two m-strata. Then $\left.\operatorname{scal}(S,-)\right|_{X^{m-2}} \leqslant 0$ for each stratum $X^{m-2}$ of dimension $m-2$.

Proof. From Thom's Isotopy Lemma ([20], [14]) it follows that $\chi_{\text {loc }}(S, P)=(-1)^{m}$ for each point $P \in X^{m-2}$. In view of Definition 1.12 we shall show that $\theta(S, P) \geqslant 1$ at almost each such point $P$. By the Normal Section Formula of [2], the density of $S$ at $P$ equals almost everywhere the density of the normal section $S_{P}:=\left(T_{P} X^{m-2}\right)^{\perp} \cap S$ at $P$. It thus suffices to show that $\theta\left(S_{P}, P\right) \geqslant 1$ almost everywhere.

Fix a point $P \in X^{m-2}$. A neighborhood $U_{P}$ of $P$ in $S_{P}$ lies in a tubular neighborhood around $X^{m-2}$ which implies that for each point $A$ in $S_{P}$ that is sufficiently close to $P$, the Euclidean distance of $A$ to $X^{m-2}$ equals the Euclidean distance between $A$ and $P$.

We denote by $S_{e}(P, r)$ the Euclidean sphere of radius $r$ around $P$. By hypothesis and Thom's Isotopy Lemma, the sets $S_{e}(P, r) \cap S_{P}$ are disjoint unions of sets 
$K_{1}(r), \ldots, K_{k}(r)$ which are homeomorphic images of circles. Their lengths will be denoted by $l_{1}(r), \ldots, l_{k}(r)$. The density of $S_{P}$ at $P$ is given by

$$
\theta\left(S_{P}, P\right)=\lim _{r \rightarrow 0} \frac{\sum_{i=1}^{k} l_{i}(r)}{2 \pi r} .
$$

This is proven in [2], it follows from easy arguments using Whitney-stratifications.

We will show that each term in this sum is at least 1 . It suffices to show this for the first one, so we claim that

$$
\lim _{r \rightarrow 0} \frac{l_{1}(r)}{2 \pi r} \geqslant 1
$$

If this is not the case, choose a real number $\theta^{\prime}$ with $\lim _{r \rightarrow 0} \frac{l_{1}(r)}{2 \pi r}<\theta^{\prime}<1$ and $\varepsilon>0$. For sufficiently small $r>0$, the following conditions will be satisfied:

a) $K_{1}(r)$ lies in a tubular neighborhood of $X^{m-2}$,

b) $0<l_{1}(r) \leqslant 2 \pi r \theta^{\prime}$.

Claim. There exist arbitrarily small $r>0$ and points $A, B \in K_{1}(r)$ with the following property: the geodesic between $A$ and $B$ contains a point $P^{\prime}$ of $X^{m-2}$.

To prove the claim, we need some topological arguments. Choose a homeomorphism $\varphi: S^{1}=\{z \in C:|z|=1\} \rightarrow K_{1}(r)$. We will extend $\varphi$ to a continuous map $\bar{\varphi}: B^{2} \rightarrow S$. To this end, fix some point $A \in K_{1}(r)$. Then $A^{\prime}:=\varphi^{-1}(A) \in S^{1}$ and each point $C^{\prime}$ of $B^{2}$ can be represented as

$$
C^{\prime}=A^{\prime}+t\left(B^{\prime}-A^{\prime}\right)
$$

with $0 \leqslant t \leqslant 1$ and $B^{\prime} \in S^{1}$. If $C^{\prime} \neq A^{\prime}$, this representation is unique.

Set $B:=\varphi\left(B^{\prime}\right)$. Denote the unique geodesic between $A$ and $B$ by $\gamma_{A, B}$. We define

$$
\bar{\varphi}\left(C^{\prime}\right):=\gamma_{A, B}\left(t d_{i}(A, B)\right) .
$$

Since in $\operatorname{CAT}(\kappa)$-spaces geodesics of length strictly smaller than $D_{k}$ depend continuously on their endpoints (see [4]), $\bar{\varphi}$ is a continuous map from $B^{2}$ to $S$.

Suppose that the claim were not fulfilled. Then $\bar{\varphi}$ is a continuous map from $B^{2}$ to $S \backslash X^{m-2}$. It follows that the loop $K_{1}(r)$ is contractible in $S \backslash X^{m-2}$. The image of $B^{2}$ under this map lies for $r$ sufficiently small in the neighborhood $U_{P}$. Hence $K_{1}(r)$ is contractible in $\left(S \backslash X^{m-2}\right) \cap U_{P}$.

On the other hand, $K_{1}(r)$ cannot be contractible in $S_{P} \backslash\{P\}$, since this set is homotopically equivalent to the union of the sets $K_{i}(r), i=1, \ldots, k$. By Thom's Isotopy Lemma, $\left(S \backslash X^{m-2}, P\right)$ is locally homeomorphic to $\left(\left(S_{P} \backslash\{P\}\right) \times \mathbb{R}^{m-2}, P\right)$. Therefore $K_{1}(r)$ cannot be contractible in $S \backslash X^{m-2}$ which is a contradiction. The claim is proved.

Next, choose a sufficiently small $r$ and points $A, B$ as in the claim. The geodesic $\gamma$ between $A$ and $B$ contains some (not necessarily unique) point $P^{\prime} \in X^{m-2}$. 
The length of the curve $K_{1}(r)$ is bounded by $2 \pi r \theta^{\prime}$. Since this curve is homeomorphic to a circle, we can join $A$ with $B$ by a curve $\beta$ of length bounded by $\pi r \theta^{\prime}$ which stays at a Euclidean distance $r$ away from $P$. By our assumptions, this curve lies in a tubular neighborhood of $X^{m-2}$ which implies that the Euclidean distance from each point of the curve to $P^{\prime}$ is at least $r$.

Take a series of points $A=P_{0}, P_{1}, \ldots, P_{k}=B$ on $\beta$ such that

$$
d_{i}\left(P_{j}, P_{j+1}\right)<\varepsilon, \quad j=0,1, \ldots, k-1
$$

and

$$
\sum_{j=0}^{k-1} d_{i}\left(P_{j}, P_{j+1}\right) \leqslant l(\beta) \leqslant \pi r \theta^{\prime}
$$

Let $\gamma_{j}$ be the geodesic between $P_{j}$ and $P^{\prime}$. We deduce from the CAT-inequality and from the triangle inequality for angles (see [4], Proposition 1.14)

$$
\pi=\angle P^{\prime}\left(\gamma_{0}, \gamma_{k}\right) \leqslant \sum_{j=0}^{k-1} \angle P^{\prime}\left(\gamma_{j}, \gamma_{j+1}\right) \leqslant \sum_{j=0}^{k-1} \angle\left(P_{j}, P^{\prime}, P_{j+1}\right)
$$

Let $l_{\kappa}(r)$ denote the length of the circle of radius $r$ in $M_{\kappa}^{2}$.

To estimate the angle $\angle\left(P_{j}, P^{\prime}, P_{j+1}\right)$ note that $d_{i}\left(P_{j}, P^{\prime}\right) \geqslant r$. It follows that this angle is not greater than the corresponding angle of a comparison triangle in $M_{\kappa}^{2}$ with side lengths $r, r, d_{i}\left(P_{j}, P_{j+1}\right)$ which is bounded by $\psi(\varepsilon) 2 \pi \frac{d_{i}\left(P_{j}, P_{j+1}\right)}{l_{\kappa}(r)}$ where $\psi(\varepsilon)$ tends to 1 for $\varepsilon \rightarrow 0$ (just take a Taylor development of the $\kappa$-cosine).

It follows that

$$
\pi=\angle P^{\prime}\left(\gamma_{0}, \gamma_{k}\right) \leqslant \sum_{j=0}^{k-1} \angle\left(P_{j}, P^{\prime}, P_{j+1}\right) \leqslant \psi(\varepsilon) \frac{2 \pi}{l_{\kappa}(r)} \sum_{j=0}^{k-1} d_{i}\left(P_{j}, P_{j+1}\right) \leqslant \psi(\varepsilon) \frac{2 \pi r}{l_{\kappa}(r)} \pi \theta^{\prime}
$$

If we let $\varepsilon$ tend to 0 , we get

$$
\pi \leqslant \frac{2 \pi r}{l_{\kappa}(r)} \pi \theta^{\prime}
$$

For $r \rightarrow 0$, the right hand side tends to $\pi \theta^{\prime}<\pi$, which is a contradiction. Hence the assumption $\theta<1$ cannot be fulfilled and Proposition 3.6 is proved.

The proof of the main theorem is complete.

\section{Further remarks}

A natural conjecture would be that the main theorem remains true under the weaker condition that every stratum of codimension 1 lies on the boundary of at least two 
top dimensional strata. Surprisingly, this turns out to be false. The first part of the proof of the main theorem can be generalized to this situation, the problem lies in the codimension 2 case.

Take for instance 5 points on a 4-dimensional unit sphere and join each pair of them by a semialgebraic arc of length $\frac{2 \pi}{3}$ in such a way that these arcs do not intersect. With respect to the induced length metric, this graph is a CAT(1)-space, since every non-contractible loop has length at least $2 \pi=2 D_{1}$ (see [4], Example II 1.15). The Euclidean cone $X$ over this graph with base point the center $P$ of the sphere is a (semialgebraic) CAT(0)-space by a theorem of Berestovskii (see [4], Theorem II 3.14).

On the other hand, $\chi_{\mathrm{loc}}(X, P)=1-5+10=6$ and $\theta_{2}(X, P)=10 \frac{1}{2 \pi} \frac{2 \pi}{3}=\frac{10}{3}$ which implies $\operatorname{scal}(X,\{P\})=\frac{1}{2}+3-\frac{10}{3}=\frac{1}{6}>0$. Hence the scalar curvature measure is strictly positive at $P$.

Another remark concerns the invariance of the scalar curvature measure under isometries. As Bröcker-Kuppe and Fu have shown, each Lipschitz-Killing curvature measure is preserved under any definable isometry. The scalar curvature measure is one of these curvatures. However, there are simple examples of non-definable isometries between definable sets (for instance between a circle and an ellipse). But it is not clear if there is really a big difference between "isometric" and "definably isometric". Anyway, the next corollary shows that scalar curvature measure is preserved by any isometry:

Theorem 4.1. Let $S, \tilde{S}$ be two compact, connected, definable sets and $f: S \rightarrow \tilde{S}$ an isometry between them. Then $f$ induces an isomorphism of the scalar curvature measures, i.e. for each Borel measure $\tilde{U} \subset \tilde{S}$ we have

$$
\operatorname{scal}(\tilde{S}, \tilde{U})=\operatorname{scal}\left(S, f^{-1}(\tilde{U})\right)
$$

Proof. The idea is to use Formula (1). The scalar curvature measure on strata of codimension 1 is there expressed only using inner geometric terms: certain geodesics and angles. On $m$-strata, the scalar curvature is invariant under isometries (classical), on strata of codimension 2 as well (local Euler-characteristic and density are invariant under isometries). Furthermore, the scalar curvature measure is independent of the stratification. These ideas can be easily put together to give a proof of Theorem 4.1.

\section{References}

[1] K. Bekka, D. Trotman, Sur les propriétés métriques des espaces stratifiés. Preprint 1995.

[2] A. Bernig, Scalar curvature of definable Alexandrov spaces. Adv. Geom. 2 (2002), 29-55. MR 1880000 Zbl 01701491

[3] J. Bochnak, M. Coste, M.-F. Roy, Géométrie algébrique réelle. Springer 1987. MR 90b:14030 Zbl 0633.14016

[4] M. R. Bridson, A. Haefliger, Metric spaces of non-positive curvature. Springer 1999. MR 2000k:53038 Zbl 01385418 
[5] L. Bröcker, M. Kuppe, Integral geometry of tame sets. Geom. Dedicata 82 (2000), 285-323. MR 2002e:53113 Zbl 01547916

[6] L. Bröcker, M. Kuppe, W. Scheufler, Inner metric properties of 2-dimensional semialgebraic sets. Rev. Mat. Univ. Complut. Madrid 10 (1997), 51-78. MR 98m:53084 Zbl 0920.14030

[7] Y. Burago, M. Gromov, G. Perelman, A. D. Aleksandrov spaces with curvatures bounded below. Uspekhi Mat. Nauk 47 (1992), no. 2 (284), 3-51, 222. MR 93m:53035 Zbl 0802.53018

[8] J. Cheeger, W. Müller, R. Schrader, On the curvature of piecewise flat spaces. Comm. Math. Phys. 92 (1984), 405-454. MR 85m:53037 Zbl 0559.53028

[9] G. Comte, Densité et images polaires en géométrie sous-analytiques. Thèse de doctorat, Université de Provence 1998.

[10] M. Coste, An introduction to o-minimal geometry. Universitá di Pisa, Dipartimento di Matematica 2000.

[11] M. Coste, An introduction to semialgebraic geometry. Universitá di Pisa, Dipartimento di Matematica 2000.

[12] M. Ferrarotti, About geodesic distance on riemannian stratified spaces. Preprint 1997.

[13] J. H. G. Fu, Curvature measures of subanalytic sets. Amer. J. Math. 116 (1994), 819-880. MR 95g:32016 Zbl 0818.53091

[14] M. Goresky, R. MacPherson, Stratified Morse theory. Springer 1988. MR 90d:57039 Zbl 0639.14012

[15] M. Gromov, Metric structures for Riemannian and non-Riemannian spaces. Birkhäuser 1999. MR 2000d:53065 Zbl 0953.53002

[16] M. Kuppe, Integralgeometrie Whitney-Stratifizierter Mengen. Dissertation Münster 1999. Eprint http://wwwmath.uni-muenster.de/u/broecker

[17] K. Kurdyka, G. Raby, Densité des ensembles sous-analytiques. Ann. Inst. Fourier (Grenoble) 39 (1989), 753-771. MR 90k:32026 Zbl 0673.32015

[18] T. L. Loi, Verdier and strict Thom stratifications in o-minimal structures. Illinois J. Math. 42 (1998), 347-356. MR 99c:32058 Zbl 0909.32008

[19] G. Perelman, Spaces with curvature bounded below. In: Proceedings of the International Congress of Mathematicians, Vol. 1, 2 (Zürich, 1994), 517-525, Birkhäuser 1995. MR 97g:53055 Zbl 0838.53033

[20] R. Thom, Ensembles et morphismes stratifiés. Bull. Amer. Math. Soc. 75 (1969), 240-284. MR 39 \#970 Zbl 0197.20502

[21] L. van den Dries, Tame topology and o-minimal structures. Cambridge Univ. Press 1998. MR 99j:03001 Zbl 0953.03045

[22] L. van den Dries, C. Miller, Geometric categories and o-minimal structures. Duke Math. J. 84 (1996), 497-540. MR 97i:32008 Zbl 0889.03025

[23] H. Weyl, On the volume of tubes. Am. J. Math. 61 (1939), 461-472. Zbl 0021.35503

Received 23 July, 2001; revised 22 January, 2002

A. Bernig, Institut für Mathematik, Universität Zürich, Winterthurerstr. 190, CH-8057 Zürich, Switzerland

Email: bernig@math.unizh.ch 